\title{
Gestión contralora de Proyectos Educativos Integrales Comunitarios en Instituciones Educativas
}

\author{
Romero Parra, Nelson* \\ Romero Parra, Rosario Mireya** \\ Basanta Zamudio, Gustavo José ${ }^{\star \star \star}$ \\ Romero Parra, Bethania ****
}

\begin{abstract}
Resumen
El objetivo de la investigación es analizar la gestión contralora en los Proyectos Educativos Integrales Comunitarios (PEIC). El estudio fue descriptivo con un diseño no-experimental, de campo y transeccional. Utilizándose, para la recolección de datos la técnica de la encuesta con un cuestionario. Los resultados permiten señalar, en forma general, la gestión contralora de los PEIC en las Escuelas Bolivarianas del Municipio Miranda del Estado Zulia, es ineficiente, en todas sus dimensiones: organización administrativa, fases y control, atendiendo la opinión de los Docentes y los Miembros de la Comunidad, mientras según los directivos es eficiente en todos sus aspectos.
\end{abstract}

Palabras clave: Gestión contralora, PEIC, organización administrativa, fases y control.

Recibido: 14-11-07. Aceptado: 14-04-08

* Director. Unidad Educativa Nacional "Lisandro Faría”. Licenciado en Educación Mención Biología y Química LUZ. Maestría en Gerencia Educativa URBE.

E-mail: nelsonromerop@hotmail.com

** Profesora Asociada. Universidad Nacional Experimental "Rafael María Baralt". Licenciada en Educación. Premio Cum Laude. M.Sc. Docencia para Educación Superior UNERMB. Doctora en Ciencias de la Educación. URBE. E-mail: rosarioromero5@hotmail.com

*** Profesor Instructor. Universidad Nacional Experimental "Rafael María Baralt". Licenciado en Comunicación Social LUZ. Premio Summa Cum Laude LUZ. M.Sc. en Gerencia Educativa URBE. E-mail: gustavobasanta@hotmail.com. Autor de correspondencia.

**** Profesora Instructora. Universidad Nacional Experimental "Rafael María Baralt". Licenciada en Educación. M.Sc. en Gerencia Educativa URBE. E-mail: rbetha3@hotmail.com 


\title{
Controlling Management for Integral Community Educational Projects in Educational Institutions
}

\begin{abstract}
The purpose of this study was to analyze controlling administration in Integral Community Educational Projects (ICEP). The study was descriptive with a non-experimental, transectional field design. The survey with a questionnaire used for gathering data. In a general way, results indicated that the controlling administration of ICEP at Bolivarian Schools in the Municipality of Miranda, Zulia, is inefficient in all its dimensions: administrative organization, phases and control, according to the opinion of teachers and community members, while according to the directors, it is efficient in all aspects.
\end{abstract}

Key words: Controlling management, ICEP, administrative organization, phases and control.

\section{Introducción}

La educación es en esencia y por excelencia, el proceso mediante el cual, se transmite saber y cultura a las generaciones más jóvenes, por lo que sus deficiencias, así como atributo, inciden de una u otra manera en la formación de los ciudadanos, y consecuentemente, implica diseñar y construir la sociedad que a mediano y largo plazo será la destinataria de cada individuo, mujer y hombre, de su sistema.

Ciertamente, la gran idea es entonces contextualizar el aprendizaje, lo cual significa enseñar y exigir a los alumnos de acuerdo a su contexto, donde los docentes, así como cada uno de los miembros de la gran comunidad educativa, asuman sus roles y funciones, siendo precisamente este el contexto donde cobra valor y forma el Proyecto Educativo Comunitario Integral, el cual se concibe como un proceso integral, global y permanente de construcción colectiva, porque se busca a través de éste, la construcción y dinamicidad de la vida de las instituciones educativas, a partir de su identidad elaborando, desarrollando y consolidando de manera progresiva y continua su visión y misión organizacional.

Precisamente, este ha sido el tema seleccionado para desarrollar el presente artículo de investigación, el cual tiene como objetivo analizar la Gestión ContraIora en los Proyectos Educativos Integrales Comunitarios (PEIC), en las Escuelas Bolivarianas de las Parroquias Altagracia y Ana María Campos del Municipio Miranda del Estado Zulia. La investigación fue de tipo descriptivo con diseño transaccional y modalidad de campo. Se seleccionó como población tres grupos: el primero de directivos estuvo constituido por ocho (8) directores y subdirectores; el segundo, por sesenta y cinco (65) docentes y el tercero por setecientos cuarenta y seis (746) miembros de la comunidad de las Escuelas Bolivarianas de las Parroquias Altagracia y Ana María Campos del Municipio Miranda del Estado Zulia.

Asimismo, el estudio a través de los resultados, pondrá en evidencia las debilidades que presentan las comunidades 
Gestión contralora de Proyectos Educativos Integrales Comunitarios...

Romero, Nelson; Romero, Rosario; Basanta, Gustavo y Romero, Bethania

educativas adscritas a las Escuelas Bolivarianas del Municipio Miranda, concretamente de las Parroquias Altagracia y Ana María Campos en el desarrollo de la gestión contralora.

Además, se considera importante el artículo, porque brindará en primer término, reflexiones y análisis en torno a la aplicación de una función esencialmente gerencial como lo es la gestión contralora a herramientas del sistema educativo, en sus procesos y en sus recursos, concretándose a los miembros de la comunidad educativa de un segmento de las escuelas del Municipio Miranda (Escuelas Bolivarianas), y con énfasis en su personal directivo y docente.

\section{Gestión Contralora en el Sistema Educativo Venezolano}

El sistema educativo venezolano concede importancia en la actualidad a las necesarias reformas de las instituciones educativas, independientemente de la modalidad y nivel, pues se trata de implementar herramientas conceptuales y operativas, buscando de manera sistemática la calidad, eficiencia y efectividad del trabajo desarrollado por los miembros de la comunidad educativa, al tiempo de servir de vías válidas para recuperar aspectos de la educación significativamente dañados por el descuido u omisión, así como las debilidades evidenciadas que enfrentan las instituciones de Educación Básica Bolivariana, debido a la ausencia de estrategias gerenciales cónsonas con la realidad contextualizada.

En este contexto precisamente, se fundamenta la Gestión Contralora del Proyecto Educativo Integral Comunitario, definido por Lozano y Lara (2001), como el conjunto coherente de operaciones y acciones que permiten modificar una situación educativa inicial determinada en una situación-objetivo, caracterizada por un conjunto de factores de orden social que permiten mejorar las condiciones educativas del contexto y de la población en donde se interviene.

Asimismo, en el plano metodológico la Gestión Contralora en el proyecto educativo se convierte en la unidad más operativa del proceso de planificación, y es el cual permite modificar la realidad, esto se efectiviza a través de la generación de servicios y producción de bienes educativos cada vez más adecuados a la comunidad, por lo cual su importancia reside en la ruptura de los vínculos con el modelo prescriptivo de la planificación tradicional, pues no se limita al diagnóstico y la programación, sino incluyendo la acción colectiva.

De otro modo, la alternativa más inmediata en lo posible, consiste en establecer la Gestión Contralora en los proyectos educativos institucionales en respuesta a exigencias normativas con el carácter de un plan formal o medio más para conseguir fondos, así como una estrategia de mejoramiento y cambio institucional.

En este orden de ideas, la Gestión Contralora a través del Proyecto Educativo Integral Comunitario, parece ser una vía realmente válida en cuanto permite una mayor posibilidad de logros, en la medida en la cual se parte de especificidad de una institución y todo cuanto se realiza está en función de una identidad Institucional, en el marco de los lineamientos políticos generales (Péñate y Santiago, 2001:127). Además, son activi- 
dades de la comunidad educativa que, en tanto proceso de reflexión colectiva, otorga identidad, vigencia y continuidad a la institución, y se erige en el criterio básico a partir del cual se derivan todas las acciones.

En este contexto, el directivo ciertamente es el responsable de liderar, apoyado y asesorado por el supervisor, en este proceso de aprendizaje colectivo, capacitándose a su vez para aumentar su propia capacidad de participar con otros, de negociar, de orientar las acciones, de evaluar y realizar nuevas propuestas, y de realizar el control necesario del cumplimiento de los compromisos asumidos.

Por ello, en la actualidad no se puede concebir el cumplimiento de un objetivo sin antes planificar como lograrlo, con los pies en el presente necesariamente se debe visualizar el futuro, teniendo la flexibilidad para adecuar el plan en un determinado momento a unas determinadas circunstancias.

Asimismo, el gerente educativo para lograr la efectividad de los Proyectos Educativos Integrales Comunitarios debe establecer un proceso de control y seguimiento para corroborar el cumplimiento de las actividades, la resolución o no de problemas, logro de los objetivos planteados, de tal manera de comparar la actuación real con la prevista y marcar los desvíos para corregir la acción, o bien alterar las decisiones y planes.

En esa misma línea de pensamiento, Chiavenato (2004), plantea la Gestión Contralora es necesaria para lograr la estrategia de una institución determinada, la implementación de proyectos estratégicos implica seleccionar la combinación apropiada de supervisión y evaluación
Institucional para lograr la estrategia. No obstante, no suministra el mecanismo mediante el cual se pueda incentivar al personal para hacer funcional la estructura, y es por lo cual surge la necesidad de la Gestión Contralora; enfocada más hacia el desempeño del personal.

Por ello, desde los inicios de la planificación se ha implementado el control, pero en su forma más rígida, funcionando más como agente de castigo que como agente correctivo u orientador, ya hace varios años que en las instituciones ha tomado especial relevancia el concepto de gestión contralora, que según Rondón (2004), debe entenderse como un proceso que sirve para guiar la gestión institucional hacia los objetivos de la organización y es un instrumento para evaluarla a través de los Proyectos Educativos Integrales Comunitarios.

En este sentido, resulta importante destacar cuando se habla de la planificación, específicamente de la Gestión Contralora en la educación venezolana debe hacerse hincapié en el plan de evaluación de los Proyectos Educativos Integrales Comunitario, pues esta es la fase en la cual se efectúa el acompañamiento y evolución de los objetivos mediante monitoreos puestos en marcha previo, durante y después de la ejecución del proyecto y se mide el nivel de compromiso real observado.

Por otro lado, en el continente latinoamericano, existen diferencias importantes entre las concepciones clásica y moderna de gestión contralora, la primera diferencia observada es aquella que incluye únicamente al control operativo y lo desarrolla a través de un sistema de información relacionado con la contabilidad 
Gestión contralora de Proyectos Educativos Integrales Comunitarios...

Romero, Nelson; Romero, Rosario; Basanta, Gustavo y Romero, Bethania

de costos, mientras que la segunda, integra mucho más elementos de la supervisión y evaluación institucional, contemplando además, una continua interacción entre todos ellos.

A esto se adiciona, la cuantificación económica del valor otorgada a los conocimientos obtenidos por los estudiantes, de tal manera de evaluar los beneficios económicos posibles, y se evaluaría el desempeño de los docentes desde el punto de vista del cumplimiento de estrategias, (probablemente constructivas en el proceso enseñanza-aprendizaje). Para el logro económico de la organización, se estaría viendo a las instituciones educativas como una empresa.

En efecto, para lograr la Gestión Contralora se lleve a cabalidad, es necesario establecer los vínculos funcionales para integrar las variables técnicas-organizativas-sociales de una institución educativa, como lo establece el Proyecto de Resolución sobre la Organización y Funcionamiento de la Comunidad Educativa del Ministerio de Educación y Deportes (2005), concretamente en su artículo 3, referido a los objetivos de la Comunidad Educativa Bolivariana, entre los cuales destacan por su importancia dentro de la reforma educativa emprendida por el Gobierno Nacional:

a) Impulsar la participación protagónica y corresponsable de padres, madres, representantes, estudiantes docentes, administrativos, obreros y comunidad en general en la planificación, formación, ejecución y control de la gestión educativa. b) Ejercer la contraloría social de los programas sociales, recursos financieros y materiales, dentro de la institución educativa, así como las actividades inherentes al desarrollo del proceso educativo Bolivariano en el marco del estado docente.

Sin embargo, según Piñero (2005), en la mayoría de las instituciones educativas se observa que los Proyectos Educativos Integrales Comunitarios, cuando son desarrollados presentan muchas deficiencias y dificultades debido a la falta de un seguimiento adecuado por falta del supervisor y los directivos de las organizaciones educativas.

Precisamente, la problemática se evidencia durante cada año escolar cuando observamos que los líderes o gerentes de las escuelas bolivarianas no están asumiendo en su mayoría con claridad y ejecución gerencial, proyectos con una dimensión, la cual involucre y sensibilice a la mayoría de los miembros de la comunidad educativa y de su entorno, en consecuencia no se desarrolla una gestión contralora eficiente.

En este sentido, Rondón (2004) señala que existe una tendencia hacia el predominio de los intereses grupales y la planificación normativa, escasa coordinación y seguimiento de los proyectos comunitarios, mínimos referentes teóricos del director para manejar estrategias de gestión contralora en su ejercicio gerencial y liderazgo que en nada benefician los proyectos educativos adelantados, y en consecuencia, son mínimos los beneficios obtenidos por la institución y la comunidad en general.

Ciertamente, lo señalado permite establecer como pronóstico que la Gestión Contralora tiene su esencia o significado en el firme propósito de lo planifica- 
do en cualquier instancia se cumpla con la mayor eficiencia y eficacia, y no se desvíen los propósitos del proyecto.

Asimismo, los Proyectos Escolares Integrales Comunitarios, presentan en su concepto, elaboración y ejecución un grado de complejidad significativa, aunque relativa, pues involucra muchos factores, requeridos de una gestión contralora eficiente y presente en cada una de las fases del proyecto, de no ser así, no se le estaría dando cumplimiento a la normativa emanada del órgano rector o Ministerio de Educación, y en consecuencia se estaría violando la norma y por ende se estarían diluyendo los esfuerzos y recursos.

Partiendo de los elementos señalados para contextualizar la problemática que presentan las comunidades educativas, y a manera de Control de Pronóstico, en este trabajo se analizará la Gestión Contralora que la comunidad educativa está aplicando a los Proyectos Educativos Integral Comunitario (PEIC), en las Escuelas Bolivarianas, ubicadas en las Parroquias Altagracia y Ana María Campos del Municipio Miranda del Estado Zulia.

\section{Gestión Contralora}

La gestión contralora dentro de los modelos de gerencia más actuales, es asignada con diversos términos, pero siempre asociada a la función que debe desarrollar todo gerente o directivo de una organización en materia de control, seguimiento o supervisión.

En este orden de ideas, Drucker (2005), señala todo recurso humano en funciones gerenciales como son los directivos de las escuelas básicas, debe atender a la función contralora, definiéndola como el proceso mediante el cual un individuo, y consecuentemente, una organización, se asegura pues el sistema educativo en sus diferentes ámbitos y recursos se desempeñan con la calidad y el nivel establecido en el marco jurídico.

Esta función inherente a la gestión contralora, implica además, el desarrollo de acciones mediante la cual, se valora cualitativa y cuantitativamente el desempeño del recurso humano del sector educación, así como los procesos inherentes al sistema, haciendo para ello, uso de técnicas, métodos y herramientas, permitiendo establecer la calidad del desempeño de los docentes y el funcionamiento de las instituciones educativas.

Es importante señalar que, tanto los procesos gerenciales como las funciones inherentes a estos, encuentran un marco referencial en Venezuela para el desempeño de los actores educativos. En tal sentido, el desarrollo de la democracia participativa y protagónica consagrada en la Constitución Bolivariana de Venezuela (1999), así como en el Proyecto Educativo Nacional posee diversas manifestaciones, las cuales, según el Ministerio de Educación, Cultura y Deportes (2005), en términos educativos, son las siguientes:

- Construir la legitimidad político-social de programas y proyectos dirigidos a elevar la calidad de la educación.

- Elaboración colectiva de propuestas en función del cambio en las prácticas pedagógicas.

- Hacer pertinentes los aprendizajes.

- Flexibilizar el currículo para adecuarlo al entorno sociocultural. 
Gestión contralora de Proyectos Educativos Integrales Comunitarios...

Romero, Nelson; Romero, Rosario; Basanta, Gustavo y Romero, Bethania

- Participación de la comunidad en la gestión escolar, tal como está planteado en el anterior punto en el cual la escuela es el centro del quehacer comunitario.

Al atender estas características o claves educativas, se tiene pues una gestión contralora debe fundamentarse en procesos descentralizados 0 descongestionados en cuanto a funciones, subrayando en las acciones estratégicas ejecutadas al carácter de autonomía y participación real de los actores educativos.

Asimismo, señala el citado órgano ministerial, que el desarrollo de una nueva cultura política, dirigida a superar los déficit de legitimidad y de gobernabilidad, permite reconocer el nexo establecido por la verdadera descentralización y el federalismo, con la soberanía política y el ejercicio de la democracia directa, es decir, la participación integral de los ciudadanos en los asuntos públicos, partiendo desde su cotidianidad y el contexto local.

Por ello, todo proceso gerencial con vista a la descentralización de procesos, funciones y acciones debe dirigirse hacia la autonomía y democratización de los procesos político-administrativos en la escuela; lo cual resulta totalmente contrario a la seudo-participación y a la delegación de la soberanía conocida en la llamada democracia representativa, en el centralismo burocrático o Estado Unitario, debido a que se encuentra vinculada directamente con el sistema federal de organización del Estado, con el cual se produce una transferencia o bien, se delegan atribuciones, responsabilidades y recursos para ser administrados por la región o localidad.
En este contexto, los proyectos educativos, cobran especial significado, pues los mismos por definición son productos de la acción gerencial de los lideres educativos, al basarse, tal como lo refieren Carrillo y Rojas (2000), en un conjunto de acciones planificadas de manera colectiva por los docentes, directivos y miembros de la comunidad educativa y equiparadas con las acciones de los Proyectos Pedagógicos de Aula, las cuales se orientan a fortalecer los aciertos de la escuela y resolver los principales problemas pedagógicos de la mismas, permitiendo la generación de un clima de reflexión sobre la práctica pedagógica; la estimulación del desempeño profesional; la elevación de la eficacia de la escuela; la generación de procesos de aprendizaje organizacional, tal como se ejemplifica la elaboración de un proyecto educativo:

Es importante destacar todo proceso gerencial desarrollado por los directivos, deben atender objetivos generales precisos, los cuales son indicados por la Universidad Católica Andrés Bello (2002):

- Alumnos: Propiciar aprendizajes significativos e integrales, al relacionar "nuevos conocimientos" con los ya existentes en su estructura cognitiva y con sus experiencias, además le permite construir estrategias para aprender a aprender, describir sus propias potencialidades, ser más participativos, reflexivos y autónomos.

En la búsqueda del sentido de su propia existencia, formulando proyectos de vida, sabiendo tomar decisiones y resolviendo problemas; previendo las consecuencias de sus acciones, que en su vida activa sean felices, participativos, honestos, responsables y comprometi- 
dos con la transformación cultural, económica y social.

- Docentes: Favorecer el crecimiento profesional, al ofrecer posibilidades para su actualización, compartir experiencias con otros colegas y desarrollar su creatividad personal en la planificación y ejecución de la labor educativa.

- Institución Escolar: Elevar la eficiencia y la calidad de la escuela, promover la detección y atención de necesidades e intereses de los alumnos y de la comunidad escolar, contextualizados en el marco del Currículo Básico Nacional y del Currículo Estadal.

Asimismo, la Universidad Católica Andrés Bello (2002), indica los proyectos educativos exigen el desarrollo de acciones gerenciales al contemplar como principios la globalización, mediante el cual se organizan todos los componentes del currículo con especial énfasis en los ejes transversales; el carácter investigativo, asumiendo la investigación acción en el aula como parte esencial del proceso de construcción del aprendizaje; el carácter social, mediante el cual se busca dar respuesta desde el plano educativo en la crisis ética y moral del país.

En todo caso, lo importante de los proyectos educativos es buscar abordar las necesidades, intereses y expectativas de forma permanente y sistemática del hecho educativo y de sus actores, al señalar en este orden de ideas el Ministerio de Educación, Cultura y Deportes (2005), que se trata de un proceso de valoración continua bajo una perspectiva constructivista; que permite recoger y analizar experiencias previas, así como el desarrollo y alcances progresivo por parte de los alumnos en función de los objetivos generales de la etapa.

Por otra parte, el denominado control gerencial o gestión contralora, así como la dirección a la cual se encuentra estrechamente vinculada, es una acción de carácter gerencial, pues define las funciones administrativas que debe cumplir todo director de educación básica en el contexto del diseño, elaboración y ejecución de proyectos educativos diversos, y consiste según lo señala Robbins (2005), en establecer estratégicamente funciones para ejecutar actividades con el propósito de alcanzar los objetivos y metas derivadas o contenidas en la planificación, considerando para ello, recursos humanos, materiales, técnicos y financieros; mientras que el control es el ejercicio de la autoridad de mando desprendido de la dirección, consistente en atender al desarrollo, evolución y alcances de cada actividad contenida en las diferentes etapas de los planes previstos en la planificación.

Por ello, especialistas en el campo gerencial como Munch y García (1999), definen la dirección como: "... la ejecución de los planes de acuerdo con la estructura organizacional, mediante la guía de los esfuerzos del grupo u organización a través de la motivación, la comunicación y la supervisión".

Según estos autores, la importancia de la dirección reside en enmarcar todos los lineamientos establecidos durante la planificación y la organización, y pues a través de ella, se logran formas de comportamiento organizacional más deseables, siendo determinante en la moral de los miembros de la comunidad educativa y consecuentemente en su producti- 
Gestión contralora de Proyectos Educativos Integrales Comunitarios...

Romero, Nelson; Romero, Rosario; Basanta, Gustavo y Romero, Bethania

vidad, pues su calidad se refleja el logro de los objetivos, en la implantación de los métodos de organización y en la eficacia de los sistemas de control, permitiendo el establecimiento de una comunicación fluida.

\section{Los Proyectos Educativos Integrales Comunitarios}

Los proyectos educativos en un sentido global, son definidos por Lozano y Lara (2001), como una acción cultural o acción instrumental con dos grandes tendencias sobre las concepciones inherentes al mismo. La primera, entendiendo el proyecto como estrategia de carácter instrumental, perfilada como una herramienta útil para la planificación, pues favorece el logro de la eficiencia interna de las actividades y/o procesos educativos y pedagógicos, como plan de trabajo determinado y estático, orientado a la previsión de las condiciones administrativas que resulten más eficaces en periodos concretos.

La segunda concepción de los proyectos educativos, apunta hacia la definición de una estrategia cultural centrada su interés en la naturaleza del proyecto como proceso dinámico con sentido histórico, permitiendo articular en torno a éste, todo el quehacer educativo, destacando la importancia en cuanto posibilita a los actores que hacen vida en la comunidad educativa y local, reflexionar de manera crítica - deliberativa en la educación como una alternativa para la transformación de un país, de una región, de un municipio.

En el caso concreto de los Proyectos Educativos Integrales Comunitarios (PEIC), son en definición del Ministerio de
Educación y Deportes, citado por Morales (2006), una herramienta contextualizada en la nueva escuela comunitaria "en el escenario de la institución educativa, tiene como fundamento la formación integral del sujeto-ciudadano, como artífice de su propio desarrollo, en el contexto de una democracia participativa y protagónica con equidad social".

Este tipo de proyecto educativo está cristalizado casi en toda América Latina como lo refiere la citada autora, con base en tres dimensiones características, las cuales están acompañadas de líneas prioritarias, las cuales buscan concretar como producto un ciudadano en situación de plena intervención en la gestión local.

\subsection{Organización Administrativa de los PEIC}

Según Peñate y Santiago (2001), establecen la organización administrativa y gerencial de un proyecto educativo, independientemente de su naturaleza y propósito, debe llevarse a cabo en atención a una serie de pasos o etapas, las cuales tienen como función orientar a quienes lo elaboran, aportando un método para proceder en cada uno de sus elementos constitutivos; y en tal sentido, dichos pasos se explican a continuación:

- Elaboración de la Agenda: este proceso se inicia con la convocatoria por parte de los gerentes o directivos de la institución educativa a los miembros de la comunidad educativa para la organización del Proyecto Educativo Integral Comunitario (PEIC), rigiéndose por las normas preestablecidas; o bien, mediante la elaboración de líneas de conductas regulando la vida en la escuela, así como fijan- 
do fechas limites a cada una de las actividades de los diversos procesos que se cumplirán en la adopción del proyecto.

El Ministerio de Educación, Cultura y Deportes (2003) lo asocia con: a) conformación de un equipo promotor comunitario integrado por el personal directivo, docente, alumnos, administrativo, obrero de la institución, asociación civil, fuerzas vivas y otros actores de la comunidad, b) generar estrategias orientadas a incentivar los miembros de la institución y de la comunidad hacia la participación activa, c) reunión preparatoria con los miembros de la comunidad, informantes claves, personal de la institución para el abordaje y diagnóstico de la realidad educativa y comunitaria, d) promover la creación de asambleas comunitarias para la discusión de la comunidad, desde la perspectiva que se tiene y la escuela buscada.

Este proceso busca establecer estructuras interactivas apoyadas en el compromiso y la toma de decisiones, enmarcado en acciones comunitarias que incentiven la negociación y la unificación de criterios. Estos indicadores dejan claro cualquier decisión u opción debe estar legitimada por los actores interactuantes en el hecho educativo.

- Conformación de Grupos: de forma democrática y participativa se eligen mediante un método conocido por todos, los miembros que integrarán los diferentes grupos deliberantes para que analicen las propuestas que definirán el Proyecto Educativo Integral Comunitario. En el caso particular de esta fase, el director de la escuela básica o unidad educativa debe realizar una inducción a los padres y representantes, a los educandos, a los docentes, al personal administrativo y obrero y miembros del sector productivo y representativo de la localidad o zona de influencia de la institución, destacando la responsabilidad que asumirá cada uno en la ejecución del Proyecto Educativo Integral Comunitario.

- Adopción del Proyecto: concluida la etapa de la deliberación, se procederá a consultar al Consejo Escolar, para posteriormente revisar las diversas propuestas elaboradas por todos los grupos deliberantes con el propósito de estructurar el proyecto educativo que se adoptará.

- Plan Operativo: el director de la escuela básica o unidad educativa, procederá a presentar a la comunidad educativa, dentro de un tiempo prudencial posteriores a la adopción del proyecto, el plan operativo del mismo, el cual deberá contener metas, estrategias, recursos y cronograma de actividades necesarias para alcanzar los objetivos del Proyecto Educativo Integral Comunitario (PEIC), y su evaluación.

En cuanto a la definición de este aspecto en concreto, señalan Peñate y Santiago (2001), juega un papel importante lo realizado en el planteamiento institucional, pues los diferentes elementos del uno y del otro se conjugan y se interrelacionan, de tal manera que la filosofía educativa, las metas, las estrategias, y el Currículo Básico Nacional (CBN), aspectos que deben necesariamente ser programados en función de las necesidades diagnosticadas por la comunidad educativa local.

- Modificación del Proyecto: un proyecto educativo puede, no necesariamente, ser modificado durante su ejecución, decisión que dependerá de los obstáculos o limitaciones presentadas en 
Gestión contralora de Proyectos Educativos Integrales Comunitarios...

Romero, Nelson; Romero, Rosario; Basanta, Gustavo y Romero, Bethania

materia de recursos humanos, materiales, técnicos y/o financieros; sin lo cual esto indique de manera categórica, la realización de cambios radicales.

\subsection{Fases de los PEIC}

Al abordar en concreto las fases para la construcción de los Proyectos Educativos Integrales Comunitarios, denominados PEIC, Peñate y Santiago (2001), explican la necesidad de proceder en su elaboración, contemplar en su diseño metodológico las respuestas que hipotéticamente puedan satisfacer las necesidades, intereses y expectativas de los miembros de la comunidad educativa a la que se destina el mismo como producto, así como de aquella constituida por su entorno local.

Por ello, si bien, se recomienda el análisis por anticipado las posibles respuestas que darán satisfacción en lo buscado, tampoco puede partir de supuestos a priori; señalando en tal sentido Peñate y Santiago (2001), que por el contrario, si una comunidad educativa desea que su Proyecto Integral Comunitario (PEIC), sea viable, factible concreto y evaluable, debe elaborarlo en atención a una serie de fases especificas, las cuales son descritas a continuación:

- Primera Fase. Diagnóstico del Contexto: Ios miembros deliberantes de los diversos grupos participantes en la elaboración del Proyecto Educativo Integral Comunitario deberán evaluar las necesidades o problemas significativos que ofrezca el diagnóstico elaborado en el planteamiento institucional respectivo a un año escolar, jerarquizando y dando prioridad a los aspectos más relevantes, especialmente a aquellos encontrados vinculados con la formación integral de los educandos, con sus familias y con la comunidad local.

En este orden de ideas, deberán tener en cuenta los resultados obtenidos en las evaluaciones institucionales más recientes. De estos análisis, surgirán las propuestas relacionadas con el currículo, con las estrategias empleadas por los docentes, con el uso de recursos (materiales, técnicos, instruccionales o didácticos, financieros), con las necesidades de material y con la definición de criterios de evaluación escolar, así como sobre los aspectos inherentes a la organización de proyectos pedagógicos diversos, actividades de uso del tiempo libre, de conservación del medio ambiente, y de programas de extensión a la comunidad.

Morales (2006:75) plantea que "todo gerente realiza un diagnóstico de las situaciones para apoyar el proceso de toma de decisiones porque un diagnóstico a tiempo le permite tomar las medidas necesarias y disponer los recursos pertinentes".

Por otro lado, el diagnóstico permite al gerente educativo diferenciar los síntomas y estar atento a cualquier situación que se pueda presentar, sin recurrir a la intuición, lo que se convierte en un proceso que le permite tomar decisiones en un momento determinado, seleccionando la mejor opción entre varias de ellas.

Según el Ministerio de Educación, Cultura y Deportes (2003), debe efectuarse con estrategias convencionales y no convencionales de recolección de información, y convalidar los datos a través de reuniones y trabajo en equipo, con metodología participativa. Debe retomar los si- 
guientes elementos: a) Diagnóstico de la realidad y de la realidad comunitaria, b) Análisis, jerarquización y selección de situaciones evidenciadas por grupos de actores y c) Aplicación metodológica de construcción participativa.

- Segunda Fase. Definición de Metas: explican Peñate y Santiago (2001), tan pronto como se hayan analizado las diversas propuestas, los miembros de la comunidad educativa conjuntamente con el Consejo Escolar, deberá formular de manera democrática y participativa las metas del proyecto en términos de objetivos a largo, mediano y corto plazo, las cuales deberán ser congruentes con los objetivos y metas desarrollados en el planteamiento integral del proyecto.

Así mismo, los principios y fines educativos del Proyecto Educativo Integral Comunitario deberán enmarcarse en la filosofía que ha sido elaborada, desde las necesidades mismas de formación de la comunidad escolar; tarea esta, que deberá realizarse mediante la organización de talleres que integren a los docentes, estudiantes, padres y representantes, directivos y el personal administrativo de la escuela.

Ciertamente, esta fase del PEIC, es por su naturaleza, un trabajo de reflexión que lidera el directivo de la escuela básica con la participación de todos los miembros del Consejo Escolar para ser debatido con la comunidad educativa. Por ello, es importante tener presente siempre a manera de principio, que debe utilizarse una metodología participativa, favoreciendo la discusión y definición de metas, pues con ello se garantiza en gran medida, el compromiso de todos en la ejecución de las tareas o actividades programadas.
- Tercera Fase. Marco de Referencia: este se elabora como trabajo colectivo con el propósito de señalar el enfoque pedagógico, filosófico, socio - educativo y curricular, orientando como parte del Proyecto Educativo Integral Comunitario el trabajo educativo de la escuela básica.

- Cuarta Fase. Plan de Acción Participativa: la definición de las metas permite organizar el plan de acción que debe presentar el director de la organización con las actividades que se desarrollarán para lograr lo propuesto y visualizar el cambio esperado en la formación de los educandos.

Este plan debe, según Peñate y Santiago (2001), incluir un cronograma de actividades previstas y necesarias para alcanzar los objetivos del proyecto educativo, lo que puede implicar, la elaboración de sub - proyectos por acciones, dependiendo de la magnitud de las mismas.

El plan de acción en el Proyecto Educativo Integral Comunitario (PEIC), según el Ministerio de Educación, Cultura y Deportes (2003) sistematiza acciones objetivas. La planificación tal y como se presenta en las escuelas básicas, mantiene su condición de prever las acciones objetivas que permitan viabilizar el proyecto educativo que se adelanta, de tal forma, de comprometer a una serie de actores como son el docente, el personal directivo, los representantes y los estudiantes, todos en conjunto, deben unificar esfuerzos, decisiones y acciones para consolidar los proyectos comunitarios establecidos.

- Quinta Fase. Evaluación del Proyecto: esta fase del proyecto deberá estar a cargo de los miembros del Consejo Directivo de la institución, y se realiza den- 
Gestión contralora de Proyectos Educativos Integrales Comunitarios...

Romero, Nelson; Romero, Rosario; Basanta, Gustavo y Romero, Bethania

tro del proyecto de evaluación institucional o evaluación del producto.

En todo caso, deberá tomarse en cuenta que de acuerdo con los resultados obtenidos se procederá a realizar las modificaciones que sean pertinentes según cada caso (actividades). Para ello, Peñate y Santiago (2001), recomiendan emplear los siguientes criterios de análisis:

a) La contribución del proyecto en la transformación real de la vida de la comunidad en cuanto a los objetivos propuestos.

b) Mejoramiento de los niveles de apropiación y de participación de la comunidad en el desarrollo objetivo del proyecto.

c) Mejoramiento en le sentido de la calidad del servicio educativo en cuanto a los aportes regionales para solucionar la problemática encontrada.

d) Los objetivos a largo, mediano y corto plazo definidos en el Proyecto Educativo Integral Comunitario (PEIC).

\subsection{Control de los PEIC}

Siguiendo al Ministerio de Educación y Deportes, citado por Morales (2006), el seguimiento y monitoreo de los proyectos Educativos Integrales Comunitarios (PEIC), contempla una serie de acciones resultantes relevantes para alcanzar con eficiencia y eficacia las metas y objetivos establecidos en el planteamiento institucional de la escuela.

En este orden de ideas, el seguimiento consiste en realizar apreciaciones sobre la contribución de las acciones planteadas con respecto al propósito y objetivos del proyecto Educativo Integral Comunitario; mientras el monitoreo, se concreta a través de indicadores, favoreciendo la medición del grado y calidad con la cual se está desarrollando el proyecto en cuestión.

A este respecto, se enuncian y describen los criterios generales para llevar a cabo el seguimiento y monitoreo del Proyecto Educativo Integral Comunitario:

Análisis Periódico: concretamente del plan de acción, y se lleva a cabo con el propósito de establecer el grado de cumplimiento de las actividades y objetivos que deben propender al alcance de las líneas de acción con respecto a los resultados obtenidos en el desarrollo del Proyecto Educativo Integral Comunitario.

Indicadores de Gestión: esto tiene como propósito dar concreción al seguimiento y monitoreo como parte del control en la gestión contralora. Se trata de un estadístico, permitiendo medir el alcance del proceso, así como el impacto alcanzado en el desarrollo del proyecto con respecto a las metas propuestas. Los indicadores de gestión dentro del proyecto asume características comunitarias y se orientan por estándares conformadas por indicadores, confrontando con las particularidades de gestión de la escuela y la comunidad, de tal forma, que permite recoger evidencias, confrontarlas con el estándar y tomar decisiones para emitir juicios de valor sobre las acciones logradas y por consolidar.

En tal sentido, un indicador de logro, busca facilitar según lo refiere Morales (2006), el seguimiento y monitoreo de la implementación de actividades y acciones inherentes al proyecto, así como el desempeño del mismo y de sus actores involucrados. 
- Plan de Seguimiento: éste, presenta una serie de elementos, los cuales se describen a continuación: a) se estructura sobre la base de estrategias de socialización, acompañamiento y control de procesos, b) supervisión y control periódico de la ejecución del Proyecto Educativo Integral Comunitario por parte de las autoridades del plantel, de la comunidad, del equipo promotor, del coordinador académico del Municipio Escolar y del Equipo Técnico de la División Académica, autoridades educativas del nivel central.

c) Acompañamiento y asesoría, con ajustes y retroalimentación del Proyecto Educativo Integral Comunitario (PEIC), lo cual requiere necesariamente la partición del Equipo Promotor del mismo, d) Medición de los indicadores de logros en bases a investigaciones o estrategias de diagnósticos con carácter participativos, e) Estrategias de socialización, las cuales abarcan encuentros, reuniones, asambleas de participación para el análisis de los avances y limitaciones, trabajo de las comisiones, complementariedad, sustentabilidad y viabilidad del proyecto.

\section{Resultados de la Investigación}

En la Tabla 1 se observa la variable Gestión Contralora en los Proyectos Educativos Integrales Comunitarios por dimensión, la cual logró una media aritmética de $x=1,82$ puntos mayor $a x=1,76$ puntos en el diagnóstico del contexto, siendo éste el puntaje más bajo, y el superior de $x=1,87$ puntos en el plan de acción. Con relación a los sujetos, el mayor puntaje fue obtenido por los directivos $\bar{x}=$ 2,67 puntos, obteniendo los valores menores los docentes y comunidad con medias aritméticas de $\bar{x}=1,40$ y $\bar{x}=1,38$ puntos respectivamente.

En atención a los criterios y rangos establecidos, se estimó con el valor de la media aritmética obtenida por la dimensión, que las fases de los PEIC se cumplen de manera deficiente. En cuanto a los sujetos es importante señalar según la media aritmética obtenida por los directivos la misma se desarrolla de manera eficiente, mientras según los docentes y miembros de la comunidad ésta es deficiente.

Tabla 1

Distribución de medias aritméticas de la variable Gestión Contralora en los Proyectos Educativos Integrales Comunitarios por dimensión

\begin{tabular}{lccc} 
Sujetos & $\begin{array}{c}\text { Organización } \\
\text { Administrativa } \\
\text { de los PEIC }\end{array}$ & $\begin{array}{c}\text { Fases de los } \\
\text { PEIC }\end{array}$ & $\begin{array}{c}\text { Control } \overline{\mathbf{x}} \text { Total } \\
\text { de los PEIC por } \\
\text { sujeto }\end{array}$ \\
\hline Directivos & 2,72 & 2,69 & $2,622,67$ \\
Docentes & 1,34 & 1,40 & $1,471,40$ \\
Comunidad & 1,33 & 1,38 & $1,481,39$ \\
$\overline{\mathbf{x}}$ por dimensión & $\mathbf{1 , 7 9}$ & $\mathbf{1 , 8 2}$ & $\mathbf{1 , 8 6 \mathbf { 1 , 8 2 }}$ \\
\hline
\end{tabular}

Fuente: Romero, Romero, Basanta y Romero (2007). 
Gestión contralora de Proyectos Educativos Integrales Comunitarios...

Romero, Nelson; Romero, Rosario; Basanta, Gustavo y Romero, Bethania

En la Tabla 2 se observa la dimensión organización administrativa por indicadores, la cual logró una media aritmética de $\bar{x}=79$ mayor $a \bar{x}=1,65$ puntos en la elaboración de la ayuda, siendo éste el puntaje más bajo, y el superior de $x=1,88$ puntos en el plan operativo. En cuanto a los sujetos, el mayor puntaje fue obtenido por los directivos $x=2,72$ puntos y el menos valor lo obtuvieron los docentes y los miembros de la comunidad con $\mathrm{x}=1,33$ puntos ambos.

Atendiendo los criterios y rangos establecidos, se concretó con el valor de la media aritmética obtenida por la dimensión, que la organización administrativa de los PEIC se desarrolla de manera deficiente. Con relación a los sujetos, es importante señalar que según la media aritmética obtenida por los directores la misma se desarrolla de manera eficiente, mientras que según los docentes y miembros de la comunidad ésta es deficiente.

En la Tabla 3 se observa la dimensión Fases por Indicadores, la cual logró una media aritmética de $\bar{x}=1,82$ puntos mayor $a \bar{x}=1,76$ puntos en el diagnóstico del contexto, siendo éste el puntaje más bajo, $y$ el superior de $x=1,87$ puntos en el plan de acción. Con relación a los sujetos, el mayor puntaje fue obtenido por los directivos $x=2,67$ puntos, obteniendo los valores menores los docentes y comunidad con medias aritméticas de $\mathrm{x}=1,40 \mathrm{y} x$ $=1,38$ puntos respectivamente.

En atención a los criterios y rangos establecidos, se estimó con el valor de la media aritmética obtenida por la dimensión, que las fases de los PEIC se cumplen de manera deficiente. En cuanto a los sujetos es importante señalar según la media aritmética obtenida por los directivos la misma se desarrolla de manera eficiente, mientras según los docentes y miembros de la comunidad ésta es deficiente.

En la Tabla 4 podemos observar la dimensión Control de los PEIC por indicadores, la cual obtuvo una media aritmética de $\bar{x}=1,86$ puntos mayor a $x=1,81$ puntos en indicadores de gestión, siendo éste el puntaje más bajo, y el más alto de $x=1,89$ puntos en el plan de seguimiento. Haciendo mención de los sujetos, el mayor porcentaje fue obtenido por los directivos $x=2,62$ puntos $y$ el menor valor lo

Tabla 2

Distribución de medias aritméticas la Dimensión Organización Administrativa, por indicador

\begin{tabular}{lcccccc}
\hline Indicadores & $\begin{array}{c}\text { Elaboración } \\
\text { de la } \\
\text { agenda }\end{array}$ & $\begin{array}{c}\text { Conformación } \\
\text { de grupos }\end{array}$ & $\begin{array}{c}\text { Adopción } \\
\text { del } \\
\text { proyecto }\end{array}$ & $\begin{array}{c}\text { Plan } \\
\text { operativo }\end{array}$ & $\begin{array}{c}\text { Modificación } \\
\text { del proyecto }\end{array}$ & $\begin{array}{c}\overline{\mathbf{x}} \text { total por } \\
\text { sujeto }\end{array}$ \\
\hline Directivos & 2,70 & 2,75 & 2,75 & 2,75 & 2,66 & 2,22 \\
Docentes & 1,13 & 1,26 & 1,43 & 1,46 & 1,40 & 1,33 \\
Comunidad & 1,12 & 1,27 & 1,42 & 1,45 & 1,39 & 1,33 \\
$\overline{\text { X por }}$ & $\mathbf{1 , 6 5}$ & $\mathbf{1 , 7 6}$ & $\mathbf{1 , 8 6}$ & $\mathbf{1 , 8 8}$ & $\mathbf{1 , 8 1}$ & $\mathbf{1 , 7 9}$ \\
indicador & & & & & & \\
\hline
\end{tabular}

Fuente: Romero, Romero, Basanta y Romero (2007). 
Tabla 3

Distribución de Medias Aritméticas de la Dimensión Fases de los PEIC por Indicador

\begin{tabular}{|c|c|c|c|c|c|c|}
\hline$\frac{\text { Indicadores }}{\text { Sujetos }}$ & $\begin{array}{c}\text { Diagnóstico } \\
\text { del } \\
\text { contexto }\end{array}$ & $\begin{array}{l}\text { Definición } \\
\text { de metas }\end{array}$ & $\begin{array}{l}\text { Marco de } \\
\text { referencia }\end{array}$ & $\begin{array}{l}\text { Plan de } \\
\text { acción }\end{array}$ & $\begin{array}{l}\text { Evaluación } \\
\text { del proyecto }\end{array}$ & $\begin{array}{c}\overline{\mathbf{x}} \text { total } \\
\text { por sujeto }\end{array}$ \\
\hline Directivos & 2,70 & 2,70 & 2,70 & 2,62 & 2,66 & 2,67 \\
\hline Docentes & 1,27 & 1,43 & 1,37 & 1,51 & 1,40 & 1,40 \\
\hline Comunidad & 1,27 & 1,41 & 1,36 & 1,48 & 1,39 & 1,38 \\
\hline $\begin{array}{l}\bar{x} \text { por } \\
\text { indicador }\end{array}$ & 1,76 & 1,84 & 1,81 & 1,87 & 1,82 & 1,82 \\
\hline
\end{tabular}

Fuente: Romero, Romero, Basanta y Romero (2007).

Tabla 4

Distribución de Medias Aritméticas de la Dimensión Control de los PEIC

\begin{tabular}{lcccc}
\hline Sujetos & $\begin{array}{c}\text { Análisis } \\
\text { periódico }\end{array}$ & $\begin{array}{c}\text { Indicadores } \\
\text { de gestión }\end{array}$ & $\begin{array}{c}\text { Plan de } \\
\text { seguimiento }\end{array}$ & $\begin{array}{c}\overline{\mathbf{x}} \text { total } \\
\text { por sujeto }\end{array}$ \\
\hline Directivos & 2,58 & 2,62 & 2,66 & 2,62 \\
Docentes & 1,51 & 1,41 & 1,48 & 1,47 \\
Comunidad & 1,52 & 1,41 & 1,52 & 1,48 \\
$\overline{\mathbf{x}}$ por indicador & $\mathbf{1 , 8 7}$ & $\mathbf{1 , 8 1}$ & $\mathbf{1 , 8 9}$ & $\mathbf{1 , 8 6}$ \\
\hline
\end{tabular}

Fuente: Romero, Romero, Basanta y Romero (2007).

obtuvieron los docentes y miembros de la comunidad con $\bar{x}=1,47$ y $\bar{x}=1,48$ puntos respectivamente.

Cuando comparamos con los criterios y rangos establecidos, se concretó con el valor de la media aritmética obtenida por la dimensión, que el control de los PEIC se lleva a cabo de manera deficiente. En atención a los sujetos, es importante destacar según la media aritmética obtenida por los directores la misma se desarrolla de manera eficiente, mientras según los docentes y miembros de la comunidad ésta se está realizando de una forma deficiente.

\section{Conclusiones}

Luego de realizar el análisis y discusión de los resultados y tomando en cuenta los objetivos de la investigación, se llegó a las siguientes conclusiones:

a) La organización administrativa de la gestión contralora de los PEIC en las Escuelas Bolivarianas del Municipio Miranda del Estado Zulia, es ineficiente, según los Docentes y Miembros de la Comunidad. Presentando debilidades en todos sus aspectos, las cuales se evidencian en la falta de promoción de cursos de inducción para la comunidad educativa y 
Gestión contralora de Proyectos Educativos Integrales Comunitarios...

Romero, Nelson; Romero, Rosario; Basanta, Gustavo y Romero, Bethania

la falta de convocatoria a representantes de la comunidad local para que participen en dichos cursos; la falta de consulta al Consejo Escolar finalizado el proceso de deliberación, a lo que se adiciona la no revisión de propuestas; la no presentación del plan operativo a la comunidad en tiempo prudencial.

Sin embargo, atendiendo la opinión de los Directivos, la organización administrativa de la gestión contralora de los PEIC en las Escuelas Bolivarianas del Municipio Miranda del Estado Zulia, es eficiente en todos sus aspectos.

b) Las fases de la gestión contralora de los PEIC en las Escuelas Bolivarianas del Municipio Miranda del Estado Zulia, se desarrollan ineficientemente, según los Docentes y Miembros de la Comunidad. Concretamente en la prioridad dada al diagnóstico colectivo de los aspectos vinculados con la formación integral de los educandos y la elaboración de la filosofía del Proyecto Educativo Integral Comunitario, considerando las necesidades de formación integral.

Igualmente, se detectaron ineficiencias en torno a la evaluación de problemas institucionales por parte de los grupos deliberantes, así como en la formulación de sub-proyectos como parte del plan, así como el hecho de que nunca los Consejos Directivos evalúan los logros del PEIC. Por otro lado, según los Directivos las fases de la gestión contralora de los PEIC en las Escuelas Bolivarianas del Municipio Miranda del Estado Zulia, se desarrollan eficientemente.

c) El control en la gestión contralora de los PEIC en las Escuelas Bolivarianas del Municipio Miranda del Estado Zulia, es ineficiente, según los Docentes y
Miembros de la Comunidad, pues no se utilizan estrategias de socialización y acompañamiento, descuidando lo inherente a la supervisión periódica con representantes de la comunidad, dejando igualmente de lado, el uso de indicadores de gestión para la medición de logros y la autoevaluación del desempeño; en otras palabras, no se hace seguimiento a los proyectos, ni se analizan los avances en comisiones de trabajo, por lo que no hay retroalimentación.

Por otra parte, según los Directivos el control de la gestión contralora de los PEIC en las Escuelas Bolivarianas del Municipio Miranda del Estado Zulia, es eficiente en todos sus aspectos.

Finalmente se concluye, en forma general, la gestión contralora de los PEIC en las Escuelas Bolivarianas del Municipio Miranda del Estado Zulia, es ineficiente, en todas sus dimensiones: organización administrativa, fases y control, atendiendo la opinión de los Docentes y los Miembros de la Comunidad, mientras según los directivos es eficiente en todos sus aspectos.

\section{Referencias Bibliográficas}

Carrillo, G y Rojas, B. (2000). Los Proyectos Educativos. Caracas. Instituto Internacional de Andragogía.

Chiavenato, Idalberto (2004). La Administración del Recurso Humano. México. Editorial Mc Graw Hill.

Chávez, Nilda (2004). Introducción a la Metodología Educativa. Maracaibo. Talleres de Artes Gráfica.

Drucker, Peter (2005). Gerencia para el Futuro. El Decenio de los 90 y más allá. Colombia. Grupo Editorial Noriega. 
Lozano, Luis y Lara, Carlos (2001). Paradigmas y Tendencias de los Proyectos Educativos Institucionales. Una Visión Evaluativa. Santa fe de Bogotá-Colombia. Cooperativa Editorial Magisterio, Editorial Delfín Ltda. 2da edición.

Ministerio de Educación (2003). Proyecto Educativo Nacional. Caracas.

Ministerio de Educación y Deportes (2005). Proyecto de Resolución sobre la Organización y Funcionamiento de la Comunidad Educativa. Caracas.

Morales, Marisela (2006). Orientaciones para la Construcción del Proyecto Educativo Integral Comunitario (PEIC). Maracaibo. Ministerio de Educación y Deportes. Zona Educativa Zulia. División Académica. Coordinación de Formación Permanente.

Munich, Galindo y García, Martínez (1999). Fundamentos de Administración. México. Editorial Trillas.
Peñate, Montes y Santiago, Hemel (2001). Administración de Instituciones Educativas. Desde la Perspectiva del P.E.I. Santa fe de Bogotá-Colombia. Cooperativa Editorial Magisterio. Editorial Delfín Ltda. 2da edición.

Piñero, Lorena (2005). Lineamientos para la elaboración de los Proyectos Educativos. Trabajo de Ascenso. Universidad Nacional Experimental Rafael María Baralt.

Robbins, Stephen (2005). Comportamiento Organizacional. México. Pretince Hall, S.A.

Rondon, Lenis (2004). Proyecto Educativo Integral Comunitario y la Efectividad de las Funciones Gerenciales del Director en la I y II Etapas de Educación Básica. (Tesis de Maestría). Maracaibo. Universidad Rafael Belloso Chacín.

Universidad Católica Andrés Bello (2002). Doce Propuestas Para el Sistema Educativo Venezolano. Caracas. Publicaciones de la UCAB. 